\title{
Nonlinear Pricing of Shareable Products
}

\author{
Thomas A. Weber \\ École Polytechnique Fédérale de Lausanne \\ thomas.weber@epfl.ch
}

\begin{abstract}
We consider a durable-goods monopolist who is able to control the collaborative consumption of its goods on an aftermarket by a sharing tariff. Consumers are heterogeneous with respect to their respective need propensities in each period. We show that the firm may be able to extract this private information by offering a nonlinear pricing scheme, which amounts to a menu of options that distinguish themselves by different combinations of retail price and sharing tariff, whereby the latter is charged to owners at the point of sharing their item with a nonowner on the sharing market. The solution, which is obtained using optimal control theory, critically depends on the product's durability.
\end{abstract}

\section{Introduction}

A durable good may be shared with others whenever owners have no use for the product in a given time period. This gives rise to sharing markets, which have been the subject of active investigation; see, e.g., $[3,4,5$, $6,16,17]$. By retaining some form of aftermarket control over who uses a given item, as is now commonplace for numerous products (such as long-term transportation tickets, software subscriptions, studio equipment, phones, or computers), the original seller (or manufacturer) can in principle charge the original buyer ex post for usage transitions, including sharing "smart products" with others [19]. ${ }^{1}$ In this paper, we examine how shareability control can be used for the purpose of nonlinear pricing (screening), even if the need-contingent use value is perfectly known and homogenous. The idea is that agents' propensities for having a high need for the product in any given period are heterogeneous. The firm can capitalize on this ex-ante need heterogeneity and devise a menu of selling contracts which offer different retail prices and sharing tariffs, adapted to the consumer population.

The remarkable insight from our analysis is that largely independent of the specific distribution of consumer types the optimal retail-price schedule is quadratic and decreasing while the sharing-tariff schedule is linear and increasing in the consumers' subjective likelihood of need. That means that someone who is likely to need the item in the future will have to pay a lower retail price in return for a larger ex-post payment to the firm for the right to share the item with others should the opportunity arise (whenever the item is not needed). Our analysis examines the consumers' and the firm's infinite-horizon dynamic decision problems in steady state using optimal control theory (for the firm's screening problem) and standard dynamic programming techniques applied to discrete-time Markovian systems (for the consumers' respective choice problems).

\subsection{Literature}

Sharing markets allowing for mutual insurance among consumers, whose payoffs depends on the realization of their uncertain needs, much in the spirit of Arrow (1953) [2]. These aftermarkets help consumers adapt their investedness in the product, in the face of a binary decision between ownership and nonownership whenever they are not already owners whose product is still in good working condition. While manufacturers have largely come to accept the existence of sharing markets, a widescale adjustment of product design and pricing, which can be viewed as a second-order effect of sharing markets, has not yet taken place. New models for effective surplus extraction need to be found, where the firm can participate over a longer time horizon in the extraction of the unfolding consumer utility. For this a control of shareability is necessary. Such aftermarket control had been previously contemplated by firms mainly through the active design of compatibility as well as complements, resulting in proprietary product ecosystems that lock in consumers; [1] marks a beginning of that literature. Controlling the shareability of an item means to offer a one-time 'license to share' at the time when the current owner has a low need for the item. Using a standard model, [19] determines the optimal fixed sharing tariff to charge consumers for the right to share perfectly durable products. Here, we examine the con-

\footnotetext{
${ }^{1}$ The technological limitations of effective sharing control are receding rapidly for almost all durable goods, thus persistently expanding the scope of this paper to a wide variety of products. Most limits of aftermarket sharing control thus far may be due to cost (e.g., a fingerprint reader or retinal scan to unlock a drone) but are usually not fundamentally related to infeasibilty.

URI: https://hdl.handle.net/10125/64479

978-0-9981331-3-3

(CC BY-NC-ND 4.0) 
struction of a nonlinear pricing model given any level of durability. The idea of nonlinear pricing goes back to the seminal contribution by Mirrlees (1971) [10] on optimal taxation. Mussa and Rosen (1978) [11] adapted these findings for second-degree price discrimination of a continuum of consumer types. Our approach follows the general model in [18], but includes a more detailed discussion on how to determine the boundary values for the firm's optimal schedule, as well as the robustness. To the best of our knowledge, the application of nonlinear pricing to the specifics of sharing markets is entirely new.

\subsection{Outline}

The remainder of this paper is organized as follows. Sec. 2 describes the basic setup of the infinite-horizon dynamic consumer choice, as well as the firm's screening problem. Sec. 3 provides a complete set of optimality conditions which are used to characterize the firm's best nonlinear pricing schedule. Robustness and identfication issues are discussed. Sec. 4 discusses some implications for product design. We discuss our findings in Sec. 5 and provide directions for further research.

\section{Model}

We consider a dynamic economy with a continuum of infinitely lived consumers (also referred to as "agents"). Without loss of generality, the total number of agents is normalized to 1 . This number can be scaled up as needed without affecting any of the results in this paper. At the beginning of each period $t \in\{0,1, \ldots\}$, a consumer of type $\theta \in \Theta=[0,1]$ observes the realization of his random binary need state $\tilde{s}_{t}(\theta) \in\{0,1\}$ for a given durable good (e.g., a power tool, a computer, a piece of software, or a car), which is distributed so that

$$
\mathbb{P}\left(\tilde{s}_{t}(\theta)=1\right)=\theta,
$$

for all $\theta \in \Theta$, without any correlation across periods. When the need state is high (i.e., $s_{t}(\theta)=1$ ), the agent has a consumption utility of $\nu>0$ for the item; on the other hand, when the need state is low (i.e., $s_{t}=0$ ), his consumption utility in the current period is zero. To focus our attention on the sharing-related type-attribute $\theta$, we assume that $\nu$ is identical across all consumers and common knowledge, and that the consumer types $\theta$ are distributed on the type space $\Theta$ according to the smooth cumulative distribution function (cdf) $F: \Theta \rightarrow[0,1]$, such that its derivative $f=F^{\prime}$ is increasing on $\Theta$. This means that almost all types are available with positive probability density. In general, the durability $q \in[0,1]$ of the firm's product is not necessarily perfect. As in
Razeghian and Weber (2019) [14], the product fails in any given period with probability $1-q$. Hence, for $q=0$ the good becomes completely disposable, while for $q=1$ it never breaks. We denote by $g(\theta)$ the (nonnormalized) density of potential buyers which naturally depends on the product's durability $q$ as well as (multiplicatively) on the (normalized) density $f(\theta)$ of consumer types $\theta \in \Theta$. An explicit expression of this steady-state density is provided in Sec. 2.3.

\subsection{Consumer Choice}

When his need state is high, an agent of type $\theta$ can get access to the product by either purchasing it from a monopolistic firm at the retail price $r$ or borrow it on a peer-to-peer market at the sharing price $p$. Once the agent becomes an owner by purchasing the item, he remains an owner until the item breaks, which-as long as $q<1$-happens with probability 1 at some finite time in the future. As an owner, the agent can use the item when in need or rent it out on the sharing market for a net revenue of $p-\tau$, where the "sharing tariff" $\tau$ is imposed by the firm for authorizing the transfer of usership for one period, as in [17]. Hence, any agent can find himself in four possible states, being either an owner or a nonowner, and in either a high-need or a lowneed state. The per-period discount factor for all agents is $\delta \in(0,1) .^{2}$ With a functioning sharing market, it is clear that agents will consider acquiring ownership of the product only if they are nonowners in a high-need state. In that state, some agents may still prefer renting the item from the sharing market rather than committing to ownership. Let

$$
\hat{p}(\theta) \triangleq p-\tau(\theta),
$$

denote the effective transaction price for lenders in the sharing market. With this, we consider first nonowners and then owners.

Nonowners. The (discounted) value for nonowners in the low-need state is

$$
U_{0}=0+\delta\left(\theta U_{1}+(1-\theta) U_{0}\right),
$$

while for nonowners in the high-need state it is

$$
U_{1}=\max \{A, B\},
$$

where the agent chooses between the expected nonownership value,

$$
A=\nu-p+\delta\left(\theta U_{1}+(1-\theta) U_{0}\right),
$$

obtained via access on the sharing market, and the expected ownership value,

$B=\nu-r+\delta(1-q)\left(\theta U_{1}+(1-\theta) U_{0}\right)+\delta q\left(\theta V_{1}+(1-\theta) V_{0}\right)$,

obtained via buying the product from the retailer. Naturally, all of the payoffs (i.e., $U_{0}, U_{1}, A, B$ ) depend on the consumer type $\theta$.

\footnotetext{
${ }^{2}$ The discount factor is related to the per-period discount rate $d>-1$, as $\delta=1 /(1+d)$.
} 
Owners. The (discounted) value for owners in the low-need state is

$V_{0}=\hat{p}+\delta(1-q)\left(\theta U_{1}+(1-\theta) U_{0}\right)+\delta q\left(\theta V_{1}+(1-\theta) V_{0}\right)$

whereas in the high-need state it becomes

$V_{1}=\nu+\delta(1-q)\left(\theta U_{1}+(1-\theta) U_{0}\right)+\delta q\left(\theta V_{1}+(1-\theta) V_{0}\right)$

The ownership payoffs (i.e., $V_{0}, V_{1}$ ) depend on the consumer type $\theta$.

Solving the last four equations, it is straightforward to obtain the consumers' state-contingent payoffs in a stationary regime. Important for the firm's problem are the nonowner's payoffs in the high-need state, as the nonowners are the only agents who would be candidates for purchasing the product.

Lemma 1. Consider a stationary regime in the sharing economy. Nonowners in the high-need state obtain the expected payoff $U_{1}=\max \{A, B\}$, with

$$
A=\left(1+\frac{\delta \theta}{1-\delta}\right)(\nu-p)
$$

when choosing product access via the sharing market, and

$$
B=\nu-r+\frac{\delta\left(\theta \nu+(1-\theta) \hat{p}-\frac{(1-q)(\theta r+(1-\theta) \hat{p})}{1-(1-\theta) \delta q}\right)}{1-\delta},
$$

when choosing to purchase the product.

The preceding result shows that a lack of durability reduces the ownership payoffs, in the extreme to

$$
\left.B\right|_{q=0}=\left(1+\frac{\delta \theta}{1-\delta}\right)(\nu-r)=\left(\frac{\nu-r}{\nu-p}\right) A .
$$

That is, provided that $r=p$ (which is the only equilibrium when $q=0$ ) any consumer's payoff from a completely disposable product equals the payoff of not purchasing the product at all. On the other hand, a perfectly durable product provides the ownership payoff of

$$
\left.B\right|_{q=1}=\nu-r+\frac{\delta}{1-\delta}(\theta \nu+(1-\theta) \hat{p}) .
$$

Thus, the value of perfect durability is obtained as the difference between these two values:

$$
\left.B\right|_{q=1}-\left.B\right|_{q=0}=\frac{\delta}{1-\delta}(\theta r+(1-\theta) \hat{p}) .
$$

High likelihood types prefer durable products because they avoid having to purchase the item again, whereas low likelihood types expect extra payoff from being able to rent out a perfectly durable item on the sharing market in any low-need state in the future.

\subsection{Screening Problem}

To extract the type information from the otherwise anonymous consumers, the firm uses a screening mechanism $\hat{\mathbf{M}}=(\hat{\mathcal{M}}, \hat{\alpha})$, where $\hat{\mathcal{M}}$ is a message space and $\hat{\alpha}=(\hat{r}, \hat{\tau}): \hat{\mathcal{M}} \rightarrow \mathbb{R}_{+}^{2}$ is an allocation function which maps any message $m \in \hat{\mathcal{M}}$ a given agent sends to a tuple $\hat{\alpha}(m)=(\hat{r}(m), \hat{\tau}(m))$, which means that the agent pays the retail price $\hat{r}(m)$ and accepts the sharing tariff $\hat{\tau}(m)$ for future use transfers. We assume that the firm, which is also referred to as the "principal" in the context of the problem of designing its mechanism, can commit to $\hat{\mathbf{M}}$. That is, consumers do not have to worry about the firm's not fulfilling its pre-agreed obligations ex post, for example by renegotiating the sales contract based on the revealed information. The revelation principle $[9,12]$ allows the principal, without any loss of generality, to restrict attention to so-called direct revelation mechanisms $\mathbf{M}=(\mathcal{M}, \alpha)$, where the message space $\mathcal{M}$ is equal to the type space $\Theta$ and all agents announce their types truthfully. This principle holds because any agent of type $\theta$, when maximizing the payoff $B$ from buying the product under the (indirect) mechanism $\hat{\mathbf{M}}$ would solve ${ }^{3}$

$\max _{m \in \mathcal{M}}\left\{-\frac{\delta\left((1-\theta) \hat{\tau}(m)+\frac{(1-q)(\theta \hat{r}(m)-(1-\theta) \hat{\tau}(m))}{1-(1-\theta) \delta q}\right)}{1-\delta}-\hat{r}(m)\right\}$,

with solution $\hat{m}^{*}(\theta)$. But the same allocation can be achieved with the direct mechanism $\mathbf{M}=(\Theta, \alpha)$ with $\alpha=(r, \tau): \Theta \rightarrow \mathbb{R}_{+}^{2}$, with $r(\theta)=\hat{r}\left(\hat{m}^{*}(\theta)\right)$ and $\tau(\theta)=\hat{\tau}\left(\hat{m}^{*}(\theta)\right)$, for all $\theta \in \Theta$. Any type- $\theta$ agent participating in $\mathbf{M}$ would therefore happily report

$$
\hat{\theta}^{*}(\theta)=\theta
$$

so that $\mathbf{M}$ is indeed a direct revelation mechanism as claimed. The firm's beliefs about the distributions of types on $\Theta$ are given by the cdf $F$ introduced earlier. Its steady-state per-period profit from a given participating type $\theta$ is 4

$$
\pi(r, \tau, \theta)=\bar{y}_{1}(\theta) r+y_{0}(\theta) \tau, \quad \theta \in \Theta_{0},
$$

where $\bar{y}_{s}$ (resp., $y_{s}$ ) denotes the steady-state probabilities of a nonowner (resp., an owner) being in the need state $s \in\{0,1\}$; see Sec. 2.3. Agents participating in the principal's mechanism must expect a payoff $B$ from buying that exceeds the payoff $A$ expected when getting access to the product on the sharing market, thus leading to a type-dependent individual-rationality constraint,

$$
B \geq A .
$$

\footnotetext{
${ }^{3}$ All terms in $B$ that are constant with respect to $(\hat{r}, \hat{\tau})$ have been omitted, without any loss of generality.

${ }^{4}$ To keep things simple we restrict attention to revenues. Introducing a production-cost term does not introduce any fundamental difficulties. Our setting includes permanent transportation passes that become obsolete (because of a different transportation need) or are lost with probability $q$ from one period to another.
} 
This participation constraint defines the participation set $\Theta_{0} \subset \Theta$ for a given schedule $(r, \tau)$. To convert the principal's mechanism design problem into a tractable optimal control problem, we first note that the participation set is convex, provided natural monotonicity of the principal's schedule (implied by (IC) and (M) below).

Lemma 2. Let $r^{\prime} \leq 0 \leq \tau^{\prime}$. Without loss of generality, ${ }^{5}$ the participation set is of the form $\Theta_{0}=\left[\theta_{0}, 1\right]$, with $\left(r_{0}, \tau_{0}\right)=\left(r\left(\theta_{0}\right), \tau\left(\theta_{0}\right)\right)$ and $^{6}$

$$
\theta_{0}=\left[1-\frac{p-(1-\delta q) r_{0}}{\delta q \tau_{0}}\right]_{[0,1]},
$$

as long as $q \tau_{0}>0$. For $q=0$, it is $\theta_{0}=\mathbf{1}_{\left\{r_{0}>p\right\}}$; for $\tau_{0}=0$, it is $\theta_{0}=\mathbf{1}_{\left\{r_{0}>p /(1-\delta q)\right\}}$.

The proof of the last result follows from examining the sign of the difference $B-A$. For $q \tau_{0}>0$ it is positive, as long as the term $(p-r+(r-(1-\theta) \tau) \delta q)$ is positive. But the latter has a positive slope due to the assumed monotonicity of the principal's schedule.

The marginal type $\theta_{0}$ is fully determined by the starting point $\left(r_{0}, \tau_{0}\right)$ of the principal's schedule $(r, \tau)$. Note also that $\theta_{0}=1$ effectively corresponds to an empty participation set, as its (Lebesque-)measure vanishes in that case. La. 2 implies that whenever a type decides to purchase the firm's product, all higher types also purchase. Thus, the type space is partitioned into two disjoint convex sets of buyers $\left(\Theta_{0}\right)$ and nonbuyers $\left(\bar{\Theta}_{0}=\Theta \backslash \Theta_{0}\right)$.

The participating agents' truth-telling constraint requires the first-order necessary optimality condition for maximizing the purchase utility $B$ with respect to the announcement $\hat{\theta}$ (so as to obtain the contract $(r(\hat{\theta}), \tau(\hat{\theta}))$ to be satisfied at $\hat{\theta}=\theta$, so

$$
r^{\prime}(\theta)+\frac{\delta}{1-\delta}\left(\frac{q(1-\theta)(1-(1-\theta) \delta) \tau^{\prime}(\theta)+(1-q) \theta r^{\prime}(\theta)}{1-(1-\theta) \delta q}\right)=0,
$$

resulting in the incentive-compatibility constraint

$$
r^{\prime}(\theta)=-\left(\frac{(1-\theta) \delta q}{1-\delta q}\right) \tau^{\prime}(\theta), \quad \theta \in \Theta_{0}
$$

Using the second-order necessary optimality condition for truth-telling implies that

$$
r^{\prime \prime}(\theta)+\left(\frac{(1-\theta) \delta q}{1-\delta q}\right) \tau^{\prime \prime}(\theta) \geq 0, \quad \theta \in \Theta_{0} .
$$

On the other hand, differentiating the incentivecompatibility condition (IC) yields

$$
r^{\prime \prime}(\theta)+\left(\frac{(1-\theta) \delta q}{1-\delta q}\right) \tau^{\prime \prime}(\theta)-\left(\frac{\delta q}{1-\delta q}\right) \tau^{\prime}(\theta)=0,
$$

for all $\theta \in \Theta_{0}$, which gives the implementability constraint

$$
\tau^{\prime}(\theta) \geq 0, \quad \theta \in \Theta_{0} .
$$

That is, for the schedule $(r, \tau)$ to be implementable, the firm's sharing tariff $\tau$ needs to be nondecreasing. By virtue of (IC) the sharing-tariff schedule implies the retail-price schedule $r$ on the participation set $\Theta_{0}$, up to a constant.

Introducing the control variable

$$
u=\tau^{\prime}
$$

in the class of bounded measurable functions, we can now formulate the firm's screening problem as an optimal control problem of the form

$$
V\left(r, \tau, \theta_{0}\right)=\int_{\theta_{0}}^{1} \pi(r, \tau, \theta) d F(\theta) \longrightarrow \max _{r, \tau, \theta_{0}}
$$

subject to $r\left(\theta_{0}\right)=r_{0}, \tau\left(\theta_{0}\right)=\tau_{0}$, Eq. (1), and

$$
\begin{aligned}
r^{\prime}(\theta) & =-\left(\frac{(1-\theta) \delta q}{1-\delta q}\right) u(\theta), \\
\tau^{\prime}(\theta) & =u(\theta), \\
u(\theta) & \in[0, \rho] \\
\theta & \in\left[\theta_{0}, 1\right] .
\end{aligned}
$$

Eqs. (2) and (3) encapsulate the incentive-compatibility constraint (IC) and the definition of the control $u$ as the slope of the sharing-tariff schedule. Eq. (4) incorporates the implementability constraint $(\mathrm{M})$ by requiring a sign-semidefinite and bounded control. The positive constant $\rho$ denotes the upper bound for the control. It is necessary to apply standard optimality conditions; see Sec. 3. Finally, Eq. (5) restricts the firm's attention to types in the participation set $\Theta_{0}$.

Lemma 3. There exists a solution $\left(r^{*}, \tau^{*}, \theta_{0}^{*}\right)$ (together with an optimal control $\left.u^{*}\right)$, defined on $\Theta_{0}^{*}=\left[\theta_{0}^{*}, 1\right]$, to the optimal control problem (*) subject to Eqs. (1)-(5).

Proof. See [7, 8].

\subsection{Steady-State Sales Distribution}

In steady state, the number of potential buyers, for any given consumer type $\theta \in \Theta_{0}$, is constant. It is composed of those nonowners who are in a high need state (i.e., those who observed a realization $s_{t}(\theta)=1$ at the beginning of the current time period $t$ ). Current nonowners may have been owners (in the previous period) whose items failed (which happens with probability $1-q$ ). On the other hand they may also have been nonowners. In order to find the steady-state distribution of buyers (corresponding to the firm's sales) we need to determine the stationary distribution of the underlying Markov process with state transitions from nonowners (in low or high

\footnotetext{
${ }^{5}$ For $\theta_{0}=1$, the measure of the participation set $\Theta_{0}=\{1\}$ vanishes, so that the actual participation of the highest type $\theta=1$ is of no consequence for the firm and for the solution to its screening problem.

${ }^{6}$ We use the shorthand $[x]_{[0,1]} \triangleq \max \{0, \min \{1, x\}\}$ for the restriction of any $x \in \mathbb{R}$ to the interval $[0,1]$.
} 
need state) to owners (in low or high need state). By construction, a nonowner of type $\theta \in \Theta_{0}$ in a high need state will purchase the product and therefore transition to being an owner. The corresponding Markov transition matrix for the shifts among the four possible states is given by

$$
\mathbf{P}=\left[\begin{array}{cccc}
1-\theta & \theta & 0 & 0 \\
(1-\theta)(1-q) & \theta(1-q) & (1-\theta) q & \theta q \\
(1-\theta)(1-q) & \theta(1-q) & (1-\theta) q & \theta q \\
(1-\theta)(1-q) & \theta(1-q) & (1-\theta) q & \theta q
\end{array}\right]
$$

The stationary distribution $\mathbf{y}=\left(\bar{y}_{0}, \bar{y}_{1}, y_{0}, y_{1}\right)^{\top}$ is such that $\mathbf{y}^{\top} \mathbf{P}=\mathbf{y}^{\top}$, which yields

$$
\mathbf{y}=\left(\frac{(1-\theta)(1-q)}{1-(1-\theta) q}, \frac{\theta(1-q)}{1-(1-\theta) q}, \frac{(1-\theta) \theta q}{1-(1-\theta) q}, \frac{\theta^{2} q}{1-(1-\theta) q}\right)^{\top},
$$

as the steady-state probabilities $p_{s}$ of being a nonowner or owner in the states $s \in\{0,1\}$. As a result, the probability of a random agent in the current period of the given type $\theta \in \Theta_{0}$ to be a buyer is $\bar{y}_{1}$, and to be a lender it is $y_{0}$. For perfectly durable goods (when $q=1$ ) it is $\mathbf{y}=(0,0,1-\theta, \theta)^{\top}$, while for completely disposable products (when $q=0$ ) it is $\mathbf{y}=(1-\theta, \theta, 0,0)^{\top}$.

\section{Optimal Pricing Schedule}

\subsection{Optimality Conditions}

In order to solve the firm's variational problem $(*)$ subject to the constraints (1)-(5) we use the Pontryagin maximum principle (PMP) as necessary optimality condition $[13,15]{ }^{7}$ For this, we first introduce the Hamiltonian

$H(r, \tau, u, \theta, \psi)=\pi(r, \tau, \theta) f(\theta)-\psi_{r}\left(\frac{(1-\theta) \delta q}{1-\delta q}\right) u+\psi_{\tau} u$,

where $\psi=\left(\psi_{r}, \psi_{\tau}\right):\left[\theta_{0}, 1\right] \rightarrow \mathbb{R}^{2}$ denotes an adjoint variable which quantifies the "shadow value" of changes in the schedule $(r, \tau)$ as a function of the independent variable $\theta$ (corresponding to the consumer's type). Applying the PMP, the firm's optimal schedule $\left(r^{*}, \tau^{*}\right)$ is characterized by the following optimality conditions.

Theorem 1 (PMP). Let $\left(r^{*}, \tau^{*}, \theta_{0}^{*}\right)$, together with the optimal control $u^{*}$ be a solution to the optimal control problem (*) subject to (1)-(5). Then there exists an absolutely continuous adjoint variable $\psi=\left(\psi_{r}, \psi_{\tau}\right)$, defined on $\Theta_{0}^{*}=\left[\theta_{0}^{*}, 1\right]$, so that the following optimality conditions are satisfied:

(i) Maximality:

$$
u^{*}(\theta) \in \arg \max _{u \in[0, \rho]} H\left(r^{*}, \tau^{*}, u, \theta, \psi\right), \quad \theta \in \Theta_{0}^{*} ;
$$

(ii) Adjoint Equation:

$$
\left(\psi_{r}^{\prime}, \psi_{\tau}^{\prime}\right)=-\left(\frac{\partial H\left(r^{*}, \tau^{*}, u^{*}, \theta, \psi\right)}{\partial(r, \tau)}\right), \quad \theta \in \Theta_{0}^{*} ;
$$

(iii) Transversality:

$$
\left(\psi_{r}(1), \psi_{\tau}(1)\right)=0 \text {. }
$$

The three optimality conditions provided by Theorem 1 need to be supplemented by appropriate boundary conditions so as to fix the initial values $r_{0}^{*} \triangleq r^{*}\left(\theta_{0}^{*}\right)$ and $\tau_{0}^{*} \triangleq \tau^{*}\left(\theta_{0}^{*}\right)$, as well as an optimality condition for the optimal type threshold $\theta_{0}^{*}$. While the boundary conditions can be found based on economic viability of the sharing market in conjunction with the individual rationality in Eq. (1), the marginal type $\theta_{0}^{*}$ will be derived by subsequent global optimization with respect to the type threshold.

\subsection{Solution}

We first use Thm. 1 to derive the parametrized "shape" of the firm's optimal schedule $\left(r^{*}, \tau^{*}\right)$, and then pin down the unique solution by an initial condition for the schedule, followed by an optimal solution for the problem of finding the optimal marginal type $\theta_{0}^{*}$.

Shape $\left(\left(r^{*}, \tau^{*}\right)\right)$. Taking into account the transversality condition at the boundary $\theta=1$, the adjoint equation yields closed-form expressions for the adjoint variables:

$$
\psi_{r}(\theta)=\int_{\theta}^{1} \bar{y}_{1}(\vartheta) d F(\vartheta),
$$

and

$$
\psi_{\tau}(\theta)=\int_{\theta}^{1} y_{0}(\vartheta) d F(\vartheta),
$$

for all $\theta \in \Theta_{0}^{*}$. Because of the linearity of the Hamiltonian in $u$, the maximality condition implies that either the control is singular (when $\partial H / \partial u=0$ ) or the control is extremal (when $\partial H / \partial u \neq 0$ ). Indeed,

$$
\frac{\partial H}{\partial u}=\psi_{\tau}-\psi_{r}\left(\frac{(1-\theta) \delta q}{1-\delta q}\right)
$$

vanishes at the endpoint $\theta=1$, as $\psi_{r}(1)=\psi_{\tau}(1)=0$ by the transversality condition. Since it is also decreasing in $\theta$, it follows that $\partial H / \partial u>0$ on the interior of $\Theta_{0}^{*}$, so

$$
u^{*}(\theta)=\rho, \quad \theta \in \Theta_{0}^{*} .
$$

That is, the optimal control is nonsingular and lies on the boundary of the control-constraint set $[0, \rho]$. In particular, the sharing tariff increases linearly at the rate $\rho$.

\footnotetext{
${ }^{7}$ The boundedness of the control, as required in Eq. (4), is usually required in the proofs of standard versions of the PMP (which do not allow for impulse controls and jumps in the state variables-here $(r, \tau))[13,15]$; in our setup, boundedness of the control implies a rate constraint on the sharing tariff.
} 

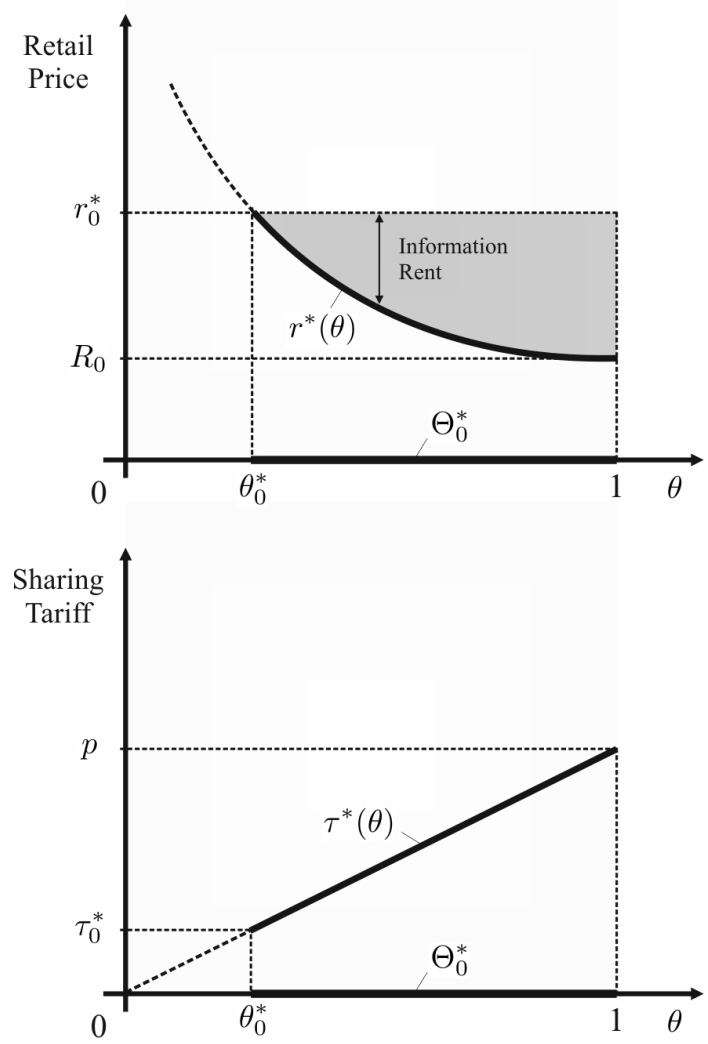

Figure 1: Optimal retail-price schedule $r^{*}(\theta)$ and sharing-tariff schedule $\tau^{*}(\theta)$ as a function of $\theta \in \Theta_{0}^{*}$.

By virtue of Eq. (2), the firm's optimal schedule $\left(r^{*}, \tau^{*}\right)$ has the components

$$
r^{*}(\theta)=r_{0}^{*}-\frac{\delta \rho q}{1-\delta q}\left(\theta-\theta_{0}^{*}-\left(\frac{\theta^{2}}{2}-\frac{\left(\theta_{0}^{*}\right)^{2}}{2}\right)\right)
$$

and

$$
\tau^{*}(\theta)=\tau_{0}^{*}+\rho\left(\theta-\theta_{0}^{*}\right),
$$

for $\theta \in \Theta_{0}^{*}$.

Initial Value $\left(\left(r_{0}^{*}, \tau_{0}^{*}\right)\right)$. The rate of increase $\rho$ is not arbitrary, as the sharing tariff is naturally bounded by the price $p$ on the sharing market, so necessarily

$$
\tau_{1}^{*} \triangleq \tau^{*}(1) \leq p .
$$

If we assume that the full sharing rent can be extracted for the highest likelihood type (for whom the no-need contingency is a zero-probability event) and set $\tau_{1}^{*}=p$, then it becomes clear that the slope $\rho$ of the optimal sharing tariff $\tau^{*}$ is bounded from above by $p$, as

$$
\rho \leq \frac{p-\tau_{0}^{*}}{1-\theta_{0}^{*}} \leq p .
$$

Thus, setting $\rho=p$ implies

$$
\tau_{0}^{*}=\tau_{1}^{*}-p\left(1-\theta_{0}^{*}\right)=p \theta_{0}^{*},
$$

(whence $\tau^{*}(\theta) \equiv p \theta$ ) and, via Eq. (1) (outside of saturation),

$$
r_{0}^{*}=\left(\frac{1-\delta q\left(1-\theta_{0}^{*}\right) \theta_{0}^{*}}{1-\delta q}\right) p \quad(>p),
$$

for all $\theta_{0}^{*} \in \Theta$. This fixes the initial value $\left(r_{0}^{*}, \tau_{0}^{*}\right)$ of the firm's optimal schedule $\left(r^{*}, \tau^{*}\right)$ as a function of the marginal type $\theta_{0}^{*}$, which is determined next.

Marginal Type $\left(\theta_{0}^{*}\right)$. The firm's profit as a function of the marginal type $\theta_{0}^{*}$ is

$$
\pi^{*}=\int_{\theta_{0}^{*}}^{1}\left(\bar{y}_{1}(\theta) r^{*}(\theta)+y_{0}(\theta) \tau^{*}(\theta)\right) d F(\theta) .
$$

Taking into account that $\partial \tau^{*} / \partial \theta_{0}^{*}=0$ and

$$
\frac{\partial r^{*}}{\partial \theta_{0}^{*}}=\frac{\delta p q \theta_{0}^{*}}{1-\delta q}
$$

it is, by virtue of Leibniz' rule and Eq. (6),

$g\left(\theta_{0}^{*}\right) \triangleq \frac{d \pi^{*}}{d \theta_{0}^{*}}=\left(\frac{\delta p q \theta_{0}^{*}}{1-\delta q}\right) \psi_{r}\left(\theta_{0}^{*}\right)-\pi\left(r_{0}^{*}, \tau_{0}^{*}, \theta_{0}^{*}\right) f\left(\theta_{0}^{*}\right)$,

for all possible marginal types $\theta_{0}^{*} \in \Theta$. Clearly, $\theta_{0}^{*}=1$ can be excluded, as it leads to zero profit for the firm. For an interior marginal type, optimality requires that the standard first-order condition holds, that is,

$$
\theta_{0}^{*} \in(0,1) \Rightarrow g\left(\theta_{0}^{*}\right)=0 .
$$

Alternatively, $\theta_{0}^{*}$ may be optimal, resulting therefore in the necessary optimality condition

$$
\theta_{0}^{*} g\left(\theta_{0}^{*}\right)=0
$$

[20] provides necessary and sufficient optimality conditions for the underlying global optimization problem on an interval, which can easily be solved numerically by simply tracking $\pi^{*}$ for $\theta_{0}^{*} \in[0,1)$, so as to choose

$$
\theta_{0}^{*} \in \arg \max _{\theta_{0} \in \Theta} \pi\left(r^{*}, \tau^{*}, \theta_{0}\right) .
$$

Main Result. The following main result summarizes our findings regarding a full characterization of the solution to the firm's screening problem.

Theorem 2 (Characterization). Let $\rho=p$. A solution $\left(r^{*}, \tau^{*}, \theta_{0}^{*}\right)$ to the principal's screening problem (*), subject to Eqs. (1)-(5), is characterized by Eqs. (8)(11) in conjunction with Eq. (13).

To compute the solution in practice, one could start by determining the adjoint variable in Eq. (6) and determine the candidates for the optimal marginal type based on the necessary optimality condition (12), so as to satisfy Eq. (13). Substituting $\theta_{0}^{*}$ in Eqs. (10) and (11) yields the missing initial values in the specification of the firm's optimal schedule in Eqs. (8) and (9); see Sec. 3.7 for an example. 


\subsection{Type-Free Schedule}

It is possible to eliminate the type parameter from the firm's optimal schedule $\left(r^{*}(\theta), \tau^{*}(\theta)\right)$ in Eqs. (8) and (9). Indeed, $\tau^{*}(\theta)=\tau$ (together with the assumption that $\rho=p$ ) implies the one-to-one mapping $\tau \mapsto \theta(\tau)$, with

$$
\theta(\tau)=\theta_{0}^{*}+\frac{\tau-\tau_{0}^{*}}{p} \in \Theta_{0}^{*}
$$

for $\tau \in\left[\tau_{0}^{*}, p\right]$, so that we obtain a "type-free" schedule $R(\tau)=r^{*}(\theta(\tau))$ of the purchase price as a function of the desired sharing premium:

$$
R(\tau)=r_{0}^{*}-\frac{\delta q}{1-\delta q}\left(1-\theta_{0}^{*}-\frac{\tau-\tau_{0}^{*}}{2 p}\right)\left(\tau-\tau_{0}^{*}\right)
$$

The type-free schedule is decreasing, as

$$
R^{\prime}(\tau)=-\frac{\delta q}{1-\delta q}\left(1-\frac{\tau}{p}\right)<0
$$

for all $\tau \in[0, p)$, and it is convex, as

$$
R^{\prime \prime}(\tau)=\frac{1}{p} \frac{\delta q}{1-\delta q}>0
$$

for all $\tau \in[0, p]$. Note that the sharing tariff $\tau$ indexes the firm's menu of contracts. More specifically, for any given $\tau \in\left[\tau_{0}^{*}, p\right]$, the contract $(R(\tau), \tau)$ is chosen by consumers of type $\theta(\tau)$. Since $\tau$-as a prospective drain on future rents - is not a desirable product attribute, its absence does constitute an attractive contract feature. More specifically, it is useful to interpret

$$
P=p-\tau \in[0, \bar{P}]
$$

as a (desirable) "premium" that comes with the shareable good, which is bounded from above by $\bar{P}=p-\tau_{0}^{*}$. The corresponding direct schedule

$$
\hat{R}(P)=R(p-P), \quad P \in[0, \bar{P}]
$$

is increasing in $P$ and concave in $P$, as one would expect in a standard nonlinear pricing scheme with quantity discounts. That means that from the firm's perspective the valuable types, i.e., those who select the contract with the highest product premium (reflected by the lowest sharing tariff), are in fact those consumers in the participation set $\Theta_{0}^{*}$ with the lowest types. The consumers with the lowest need for the item in the future must be afforded the largest product premium.

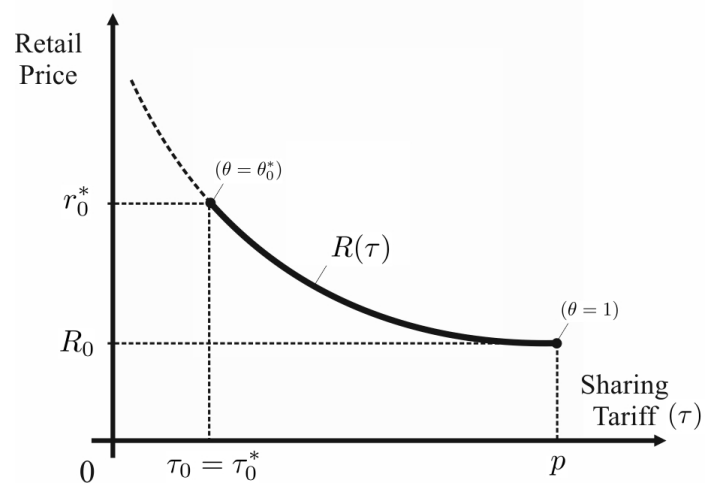

Figure 2: Type-free schedule.

\subsection{Robustness}

It is remarkable that the shape of the optimal schedule $\left(r^{*}, \tau^{*}\right)$, or-equivalently-the shape of the corresponding type-free schedule $R(\tau)$, does not depend on very detailed information about the consumers' type distribution. Indeed, only the marginal type $\theta_{0}^{*}$ (and through it the initial value $\left.\left(r_{0}^{*}, \tau_{0}^{*}\right)\right)$ depends on $F$. The cdf is used for computing the adjoint variable $\psi=$ $\left(\psi_{r}, \psi_{\tau}\right)$ in Eqs. (6) and (7), which can be interpreted as the volume of purchase and sharing transactions, respectively. Estimating the type distribution $F$, which a priori is an infinite-dimensional object, would in principle require nonparametric identification. This in turn would $a$ priori require a significant amount of data to produce an acceptable statistical fit. Thus, the company would need to gather (or procure) user-centric longitudinal information by observing customers' usage patterns for durable goods. This type of estimation may be feasible when the firm already has a significant installed base, much in contrast to a situation without significant service experience. However, the parametric form of the optimal nonlinear pricing scheme suggests that a detailed estimation of the type distribution is not really necessary, as the firm can instead focus on the much simpler task of tuning the schedule's only distribution-sensitive parameter $\left(\theta_{0}^{*}\right)$.

\subsection{Identification}

Without loss of generality, consider the firm's type-free schedule which can be written in the parametric form

$$
R(\tau)=R_{0}+a(p-\tau)+b \frac{(p-\tau)^{2}}{2}, \quad \tau \in\left[\tau_{0}, p\right],
$$

where $a$ and $b$ are nonnegative parameters, and $R_{0}$ is the product's "stand-alone" retail price when sharing is disallowed (i.e., for $\tau=p$ ). Since $R^{\prime}(p)=0$, we find that

$$
a=0 \text {. }
$$


In addition, comparing the second derivative $R^{\prime \prime}(\tau)=b$ with the result in Sec. 3.3 yields

$$
b=\frac{1}{p} \frac{\delta q}{1-\delta q} .
$$

Hence, we can rewrite the firm's type-free schedule as

$$
R(\tau)=R_{0}+\frac{1}{2}\left(\frac{\delta p q}{1-\delta q}\right)\left(1-\frac{\tau}{p}\right)^{2}, \quad \tau \in\left[\tau_{0}, p\right]
$$

where the parameters $R_{0}$ and $\tau_{0}$ need to be identified (e.g., by learning algorithms), whenever a reliable estimate of the type distribution $F$ is not available.

Remark. The value for $R_{0}$ may be fairly easy to bootstrap by observing the prices for extant non-shareable substitutes. In our model, this is the price paid by the highest likelihood type $(\theta=1)$ who has a constant need for the item. This consumer type is provided with an efficient sharing tariff $\tau=p$ extracting the full surplus from the zero-probability aftermarket transactions, and he obtains in return the largest "information rent" as surplus over his outside option,

$$
\left.[B-A]\right|_{\theta=1}=\frac{1-\delta q}{1-\delta}\left(\frac{p}{1-\delta q}-R_{0}\right),
$$

where

$$
R_{0}=R(p)=r_{0}^{*}-\frac{\delta p q}{1-\delta q} \frac{\left(1-\theta_{0}^{*}\right)^{2}}{2}
$$

so that the highest information rent becomes

$$
\left.[B-A]\right|_{\theta=1}=\frac{\delta p q}{1-\delta} \frac{1-\left(\theta_{0}^{*}\right)^{2}}{2} .
$$

\subsection{Market Clearing}

In our setting, with uniform consumption value $\nu$ across all agents, the clearing price $p \in[0, \nu]$ on the sharing market is exogenous. When the firm is a monopolist both in the primary (retail) and the secondary (sharing) market, the sharing price may be induced by the firm's optimal schedule, and naturally the firm would have an incentive to set $p=\nu$, i.e., at the highest possible value, as this guarantees maximum rent extraction from the aftermarket, without compromising retail sales. On the other hand, in steady state the sharing demand (from nonowners in the high-need state),

$$
D\left(\theta_{0}^{*}\right) \triangleq \int_{0}^{\theta_{0}^{*}} \theta d F(\theta)
$$

would need to be equal to the sharing supply (from owners in the low-need state), using Eq. (6),

$$
S\left(\theta_{0}^{*}\right) \triangleq \int_{\theta_{0}^{*}}^{1} y_{0}(\theta) d F(\theta)=\psi_{\tau}\left(\theta_{0}^{*}\right)
$$

for any $\theta_{0}^{*} \in \Theta$. The resulting market-clearing condition,

$$
D\left(\theta_{0}^{*}\right)=S\left(\theta_{0}^{*}\right),
$$

determines the marginal type $\theta_{0}^{*}$ as a function of the product durability $q$; see Sec. 3.7 for an example. We note that because of the uniform contingent use value $\nu$ across consumer types, the sharing price $p \in[0, \nu]$ cannot be determined by market clearing alone. ${ }^{8}$

\subsection{Illustration}

Consider the case of a uniform type distribution where $F(\theta)=\theta$ for all $\theta \in \Theta=[0,1] .^{9}$ Based on Eqs. (9) and (10), the sharing tariff is proportional to the consumer type and independent of $\theta_{0}^{*}: \tau^{*}(\theta) \equiv p \theta$. Given the uniformity of the type distribution (and in the absence of market-clearing considerations), it is best for the firm to be all-inclusive (i.e., $\theta_{0}^{*}=0$ ) which we illustrate for the special cases of perfectly durable products $(q=1)$ and completely disposable products $(q=0)$.

For $q=1$, the payoff-relevant steady-state probabilities become $\bar{y}_{1}=0$ (as nobody will need to purchase a product ever again) and $y_{0}=1-\theta$. Hence,

$$
\pi^{*}=p \int_{\theta_{0}^{*}}^{1}(1-\theta) \theta d \theta=\left(\frac{1-\left(3-2 \theta_{0}^{*}\right)\left(\theta_{0}^{*}\right)^{2}}{6}\right) p,
$$

which is strictly decreasing in $\theta_{0}^{*} \in(0,1)$, so that full inclusivity $\left(\theta_{0}^{*}=0\right)$ is optimal, resulting in a profit of $\pi^{*}=p / 6$-entirely from sharing authorizations.

For $q=0$, the payoff-relevant steady-state probabilities are $\bar{y}_{1}=\theta$ and $y_{0}=0$. Moreover, $r^{*}(\theta) \equiv p$, and the firm's profit,

$$
\pi^{*}=p \int_{\theta_{0}^{*}}^{1} \theta d \theta=\left(\frac{1-\left(\theta_{0}^{*}\right)^{2}}{2}\right) p,
$$

is strictly decreasing in the marginal type, so that again $\theta_{0}^{*}=0$ is optimal. This generates the profit $\pi^{*}=p / 2$-exclusively from retail.

Comparing the firm's optimal profits for $q \in\{0,1\}$ suggests that a disposable product yields a payoff that is 3 times as high, not even considering cost. However, using the market-clearing condition (15) to determine the marginal type (yielding $\theta_{0}^{*}=1 / 2$ for $q=1$, and $\theta_{0}^{*}=0$ for $q=0$ ) doubles the profit ratio to 6 .

\footnotetext{
${ }^{8}$ In practice, a given firm would need to take the sharing price $p$ as an exogenous factor, especially in situations where the sharing supply is composed of a variety of substitute products, most of which the firm has no control over.

${ }^{9}$ The per-period unit volume of retail is $\psi_{r}\left(\theta_{0}^{*}\right)=\frac{1-q}{q}\left[1-\theta_{0}^{*}-\frac{1-q}{q} \ln \left(1-\left(1-\theta_{0}^{*}\right) q\right)\right]$ for $q \in(0,1]$, and $\psi_{r}\left(\theta_{0}^{*}\right)=\left(1-\left(\theta_{0}^{*}\right)^{2}\right) / 2$ for $q=0$. The per-period unit volume of sharing licenses is $\psi_{\tau}\left(\theta_{0}^{*}\right)=\frac{\left(1-\theta_{0}^{*}\right)\left(2-\left(1+\theta_{0}^{*}\right) q\right)}{2 q}+\frac{1-q}{q^{2}} \ln \left(1-\left(1-\theta_{0}^{*}\right) q\right)$ for $q \in(0,1]$, and $\psi_{\tau}\left(\theta_{0}^{*}\right)=0$ for $q=0$.
} 


\subsection{Nonstationary Regime}

The preceding developments were limited to the regime where the different states were distributed according to the stationary distribution y; see Sec. 2.3. However, the firm may care substantially about the nonstationary behavior of the consumers, before reaching steady state. Given the initial distribution $\mathbf{y}(0)=\mathbf{y}_{0}$ at time $t=0$, the distribution at time $t>0$ is given by

$$
\mathbf{y}^{\top}(t)=\mathbf{y}_{0}^{\top} \mathbf{P}^{t} \text {. }
$$

Hence, the firm's net present value of the schedule $\left(r^{*}, \tau^{*}\right)$,

$$
\Pi^{*}\left(\mathbf{y}_{0}\right)=\int_{\theta_{0}^{*}}^{1} \sum_{t=0}^{\infty} \delta^{t}\left(\mathbf{y}_{0}^{\top} \mathbf{P}^{t}\right)\left(0, r^{*}, \tau^{*}, 0\right)^{\top} d F(\theta),
$$

depends on the initial distribution $\mathbf{y}_{0}$.

Examples. (i) Starting at the stationary distribution, $\mathbf{y}(0)=\mathbf{y}$, the firm's discounted payoff is

$$
\Pi^{*}(\mathbf{y})=\sum_{t=0}^{\infty} \delta^{t} \pi^{*}=\frac{\pi^{*}}{1-\delta},
$$

where the per-period profit $\pi^{*}$ is given by $\bar{y}_{1} r^{*}+y_{0} \tau^{*}$. (ii) For perfectly durable products $(q=1)$, there are no repeat purchases. Given the initial distribution $\mathbf{y}_{0}=$ $(1-\theta, \theta, 0,0)^{\top}$, the firm's discounted payoff is

$$
\Pi^{*}\left(\mathbf{y}_{0}\right)=\sum_{t=0}^{\infty} \delta^{t} \int_{\theta_{0}^{*}}^{1}\left(\bar{y}_{1, t} r^{*}(\theta)+y_{0, t} \tau^{*}(\theta)\right) d F(\theta),
$$

where $\bar{y}_{1, t} \triangleq \theta(1-\theta)^{t}$ and $y_{0, t} \triangleq(1-\theta)\left(1-(1-\theta)^{t}\right) .{ }^{10}$ Compared with Sec. 3.7, a substantial portion of the firm's profit now stems from retail, which in the stationary regime is not a source of benefit.

Generalized Solution. Thm. 2 generalizes to nonstationary state distributions, by replacing condition (13) for the optimal marginal type as follows:

$$
\theta_{0}^{*} \in \arg \max _{\theta_{0} \in \Theta} \hat{\pi}\left(r^{*}, \tau^{*}, \theta_{0}\right),
$$

where, for any $\theta_{0} \in \Theta$,

$$
\hat{\pi}\left(r^{*}, \tau^{*}, \theta_{0}\right) \triangleq \int_{\theta_{0}}^{1}\left(\bar{Y}_{0} r^{*}(\theta)+Y_{0} \tau^{*}(\theta)\right) d F(\theta),
$$

with $\bar{Y}_{1} \triangleq \sum_{t=0}^{\infty} \delta^{t} \bar{y}_{1, t}$ and $Y_{0} \triangleq \sum_{t=0}^{\infty} \delta^{t} y_{0, t}$.

Theorem 3 (Generalized Characterization). Under the conditions of Thm. 2, a solution $\left(r^{*}, \tau^{*}, \theta_{0}^{*}\right)$ to the problem of maximizing the principal's discounted profit $\Pi^{*}\left(\mathbf{y}_{0}\right)$ for any given initial distribution $\mathbf{y}_{0}$, subject to Eqs. (1)-(5), is characterized by Eqs. (8)-(11) in conjunction with Eq. (13').
The preceding result describes the firm's best stationary schedule, when faced with a nonstationary distribution of states starting with initial distribution $\mathbf{y}_{0}$.

\section{Implications for Product Design}

The combination of retail price and sharing tariff allows the firm to extract type-specific rents, without bunching, from all participating customers. Enabling the products technologically to detect and control usage transitions (e.g., by adding a fingerprint reader), as required for the meaningful implementation of a positive sharing tariff, may be costly; see [19]. ${ }^{11}$

La. 2 implies that the optimal monopoly price without sharing rent (i.e., for $\tau=0)$ is $r^{m}=p /(1-\delta q)$, resulting (for a stationary regime) in the per-period profit

$$
V^{m} \triangleq V\left(r^{m}, 0,0\right)=\frac{(1-q) p}{1-\delta q} \int_{0}^{1} \frac{\theta d F(\theta)}{1-(1-\theta) q} .
$$

Provided the required investment $I$ for the detection technology and a per-period cost $\gamma$ related to the monitoring and administration of usage transitions, for the sharing control to be economically viable, the following condition needs to be satisfied:

$$
I+\frac{\gamma}{1-\delta} \leq \frac{V\left(r^{*}, \tau^{*}, \theta_{0}^{*}\right)-V^{m}}{1-\delta} .
$$

The firm's monopoly profit vanishes for perfectly durable goods (i.e., when $q=1$ ), rendering active sharing authorization a strictly improving strategy in that case.

\section{Conclusion}

Heterogeneity in the agents' need propensities in conjunction with the firm's assumed capacity to control its products' shareability opens the opportunity for seconddegree price discrimination. The firm can offer a menu of options to the agents which self-select into different product offerings. Each option consists of a retail price and a sharing tariff. We have shown that for a rateconstrained sharing tariff it is optimal for the firm to provide a retail-price schedule that is quadratically decreasing in a consumer's likelihood type and a sharing-tariff schedule that is linearly increasing in the agent's likelihood type (see Fig. 1), whereby the rate of increase is such that the highest type $(\theta=1)$ is offered effectively

\footnotetext{
${ }^{10}$ At time $t>0$, the probability of not yet having bought the item over the previous $t$ periods (from 0 to $t-1$ ) is $(1-\theta)$, so that $\bar{y}_{1, t}=\theta(1-\theta)^{t}$. Summing up the purchasers of the previous periods yields (by the geometric-series formula) $\left(1-(1-\theta)^{t}\right)$, so that in the current period the fraction of owners in the low-need state becomes $y_{0, t}=(1-\theta)\left(1-(1-\theta)^{t}\right)$. Note also that, as $t \rightarrow \infty$, one obtains $\bar{y}_{1, t} \rightarrow 0$ and $y_{0, t} \rightarrow 1-\theta$, consistent with our remarks at the end of Sec. 2.3 .

${ }^{11}$ In some cases the costs may be very low, e.g., when sharing a train ticket, which requires merely an update of the usage authorization.
} 
a stand-alone product (with disabled shareability) where the sharing tariff equals the price of access $p$ in the sharing market.

Not all agents may choose to participate. The schedule of sharing tariffs then starts at a positive amount and increases linearly to the sharing price $p$. Agents with sharing propensity $\theta$ below the marginal likelihood type $\theta_{0}^{*}$ do not purchase and rather seek product access on a need-basis in the sharing market. The model can be closed, since all owners who do not need the item in the current period may rent it to nonowners in need, which determines the sharing price in equilibrium.

In the main text, we have abstracted from the possibility of user-tracking between purchases, where a type might already be known because of an earlier purchase with the company. This user-centric traceability would generically lead to an incentive to report types untruthfully, so as to retain anonymity and thus a potential for information rent in the future. Instead we assume that consumers may anonymize themselves, for example, by generating a new user account, so that the firm does not know more about repeat buyers than about first-time buyers.

Interesting extensions of the present model include serial need correlation, heterogeneity with respect to need-contingent use value $\nu$, and the possibility to vary the product durability (instead or in addition to the sharing tariff). The model as it stands illustrates the possibilities a firm gains when equipping their products with technology that allows for the detection of usage transitions; see also [19]. The present findings are robust in the sense that the basic shape of the optimal schedule is independent of the precise distribution of types; see Sec. 3.4 for details.

\section{References}

[1] AdAms, W., Brock, J.W. (1982) "Integrated Monopoly and Market Power: System Selling, Compatibility Standards, and Market Control," Quarterly Review of Economics and Business, Vol. 22, No. 4, pp. 29-42.

[2] ARrow, K.J. (1953) "Le rôle des valeurs boursières pour la répartition la meilleure des risques," Économetrie, Centre National de la Recherche Scientifique, Vol. 11, pp. 41-47. [Reprinted: "The Role of Securities in the Optimal Allocation of Risk Bearing," Review of Economic Studies, Vol. 31, No. 2, pp. 91-96, 1964.]

[3] BARdhi, F., Eckhardt, G.M. (2012) "AccessBased Consumption: The Case of Car Sharing," Journal of Consumer Research, Vol. 39, No. 4, pp. 881-898.

[4] BelK, R. (2014). "You Are What You Can Access: Sharing and Collaborative Consumption On- line," Journal of Business Research, Vol. 67, No. 8, pp. 1595-1600.

[5] Benkler, Y. (2004) "Sharing Nicely: On Shareable Goods and the Emergence of Sharing as a Modality of Economic Production," Yale Law Journal, Vol. 114, No. 2, pp. 273-358.

[6] Botsman, R., Rogers, R. (2010) What's Mine Is Yours: How Collaborative Consumption Is Changing the Way We Live, HarperCollins, London, UK.

[7] CESARI, L. (1983) Optimization - Theory and Applications: Problems with Ordinary Differential Equations, Springer, New York, NY.

[8] Filippov, A.F. (1962) "On Certain Questions in the Theory of Optimal Control," SIAM Journal on Control, Ser. A, Vol. 1, No. 1, pp. 76-84.

[9] GibBARD, A. (1973) "Manipulation of Voting Schemes: A General Result," Econometrica, Vol. 41, No. 4, pp. 587-601.

[10] Mirrlees, J.A. (1971) "An Exploration in the Theory of Optimal Income Taxation," Review of Economic Studies, Vol. 38, No. 2, pp. 175-208.

[11] Mussa, M., Rosen, S. (1978) "Monopoly and Product Quality," Journal of Economic Theory, Vol. 18, No. 2, pp. 301-317.

[12] Myerson, R.B. (1979) "Incentive Compatibility and the Bargaining Problem," Econometrica, Vol. 47, No. 1, pp. 61-74.

[13] Pontryagin, L.S., Boltyanskit, V.G., GAMKRELIDZE, R.V., MISHCHENKO, E.F. (1962) The Mathematical Theory of Optimal Processes, Wiley Interscience, New York, NY.

[14] Razeghian, M., Weber, T.A. (2019) "Strategic Durability with Sharing Markets," Sustainable Production and Consumption, Vol. 19, pp. 79-96.

[15] Weber, T.A. (2011) Optimal Control Theory with Applications in Economics, MIT Press, Cambridge, MA.

[16] WeBer, T.A. (2014) "Intermediation in a Sharing Economy: Insurance, Moral Hazard, and Rent Extraction," Journal of Management Information Systems, Vol. 31, No. 3, pp. 35-71.

[17] Weber, T.A. (2016a) "Product Pricing in a Peerto-Peer Economy," Journal of Management Information Systems, Vol. 33, No. 2, pp. 573-596.

[18] WeBer, T.A. (2016b) "Optimal Multiattribute Screening," Ural Mathematical Journal, Vol. 2, No. 2, pp. 87-107.

[19] Weber, T.A. (2017a) "Smart Products for Sharing," Journal of Management Information Systems, Vol. 34, No. 2, pp. 341-368.

[20] WeBER, T.A. (2017b) "Global Optimization on an Interval," Journal of Optimization Theory and Applications, Vol. 182, No. 2, pp. 684-705. 